Revista PSICOLOGIA, 2015, Vol. 29 (1), 23-34

\title{
Perfecionismo, perturbações emocionais e suas relações com o comportamento alimentar perturbado: Um estudo com adolescentes Portugueses
}

\author{
Leonor Oliveira1, Rita Francisco ${ }^{1}$, Rosa Novo ${ }^{1}$ \\ ${ }^{1}$ Faculdade de Psicologia, Universidade de Lisboa
}

\begin{abstract}
Resumo: 0 perfecionismo tem sido associado ao desenvolvimento de sintomatologia de perturbação alimentar, apesar de ser ainda necessário o seu escrutínio em adolescentes dos dois sexos. Pretendeu-se investigar o contributo e as relações entre o perfecionismo e a sintomatologia emocional no desenvolvimento de comportamento alimentar perturbado em ambos os sexos numa amostra de 531 adolescentes. Utilizaram-se o Eating Attitudes Test 26, Cuestionario Educativo-Clínico: Ansiedad y Depresión e a subescala de perfecionismo do Eating Disorder Inventory 2. Com recurso a regressões múltiplas hierárquicas identificou-se que a idade, dieta, perfecionismo auto-orientado e depressão contribuem para explicar significativamente $42 \%$ da variância da sintomatologia de perturbação alimentar nas raparigas. Nos rapazes o perfecionismo global e a sintomatologia de perturbação emocional contribuíram para explicar $10 \%$ da variância da sintomatologia relativa a esta perturbação. São discutidas as implicações dos fatores de risco estudados para o desenvolvimento das perturbações alimentares na adolescência e sua forma de avaliação.
\end{abstract}

Palavras-chave: Perturbações alimentares; Perfecionismo; Ansiedade; Depressão; Adolescência.

Perfectionism, emotional disturbance and its relation to eating disorder symptomatology: A study with Portuguese adolescents: Perfectionism has been associated with the development of eating disorder symptomatology, even though it is still necessary its scrutiny within adolescents of both sexes. We aimed to investigate the relations between perfectionism and emotional disturbance symptoms in the development of eating disorders symptomatology in a sample of 531 adolescents. The instruments used were the Eating Attitudes Test 26, the Cuestionario Educativo-Clínico: Ansiedad y Depresión and the perfectionism subscale of the Eating Disorder Inventory 2. By means of hierarchical multiple regression analysis was possible to identify, within the adolescent girls' group that diet, self-oriented perfectionism and depression contributed significantly for $42 \%$ of the variance of eating disorder's symptoms. In the adolescent boys' global perfectionism and emotional disturbance symptoms that contributed to explain $10 \%$ of the symptomology's variance of this disturbance. Implications for the studied eating disorders risk factors' in adolescence are discussed so as its assessment means.

Keywords: Eating disorders; Perfectionism; Anxiety; Depression; Adolescence.

As perturbações alimentares (PA) são caracterizadas por graves distúrbios do comportamento alimentar (APA, 2002) que refletem a confluência entre psicologia individual, determinantes biológicos e fatores socioculturais (e.g., Keery, van den Berg, \& Thompson, 2004). Ao nível individual, as PA têm sido relacionadas, entre outros, com fatores predisponentes como o sexo feminino, índice de massa corporal (IMC) elevado, perfecionismo (Bulik et al., 2003; Forbush, Heatherton, \& Keel, 2007), e ansiedade e/ou depressão (Vince \& Walker, 2008), sendo que a adolescência é apontada como a etapa de desenvolvimento em que se iniciam estas perturbações (e.g., Dunker \& Philippi, 2003). No entanto, as relações entre perfecionismo, sintomatologia de perturbação emocional e de perturbação alimentar não foram ainda suficientemente escrutinadas em amostras de adolescentes de ambos os sexos (especialmente em Portugal), em parte devido à escassez de estudos com rapazes, associada à menor prevalência de PA no sexo masculino (Striegel-Moore \& Bulik, 2007). Pretende-se, assim, com este estudo aprofundar as relações entre estes possíveis fatores de risco e o comportamento alimentar perturbado de adolescentes portugueses de ambos os sexos.

${ }^{1}$ Dados de contacto para correspondência: Rita Francisco, Faculdade de Psicologia, Alameda da Universidade, 1649-013 Lisboa. Email:rmfrancisco@psicologia.ulisboa. 


\section{Peso, dieta e PA}

Um IMC elevado é considerado um fator de risco para o desenvolvimento de PA em raparigas e rapazes (e.g., Babio, Canals, Pietrobelli, Peréz, \& Arija, 2009), pelo que é apontado como preditor das mesmas em adolescentes (Ferreiro, Seone, \& Senra, 2011). Alguns estudos, no entanto, não verificam esta relação de predição na população adolescente (e.g., Bento et al., 2010).

Quer se verifique devido ao excesso de peso, quer à perceção do mesmo, o comportamento de dieta, ou seja, a restrição alimentar, apesar de frequente na adolescência (Patton, 1988), é também apontado como fator de risco para o desenvolvimento de PA em ambos os sexos (e.g., Ikeda, 2001). 0 risco, porém, parece ser maior nas raparigas que efetuam dieta, mesmo quando apresentam peso normal (e.g., Daee et al., 2002), talvez porque o corpo ocupa uma dimensão central na autoavaliação das mulheres nas culturas ocidentais (Stice, Hayhard, Cameron, Killen, \& Taylor, 2000). É também na adolescência que surgem as primeiras diferenças na ingestão e seleção de alimentos: os rapazes ingerem mais calorias que as raparigas, talvez por elas experienciarem maior insatisfação com o corpo que eles (Rolls, Fedoroff, \& Guthrie, 1991), por se aperceberem mais do excesso de peso (Vaughan \& Halpern, 2010) ou por procurarem ideais corporais diferentes, o que motiva as raparigas para a magreza e os rapazes para a muscularidade (Davis, Karvinen, \& McCreary, 2005). Assim sendo, no espetro do comportamento alimentar perturbado, as raparigas apresentam mais comportamentos de dieta (Vaughan \& Halpern, 2010) e os rapazes mais excesso de exercício físico (Lewinsohn, Seeley, Moerck, \& Striegel-Moore, 2002). Não surpreendentemente, atentando aos dados apresentados acima, a prevalência de PA é mais elevada nas raparigas (e.g., Hautala et al., 2007; Smink, van Hoeken, Oldehinkel, \& Hoek, 2014).

\section{Perfecionismo e sua relação com PA}

A revisão de literatura de Franco-Paredes, Mancilla-Díaz, Vázquez-Arévalo, López-Aguilar e ÁlvarezRayón (2005) mostra a diversidade de estudos sobre perfecionismo e PA, quer em termos de resultados acerca da relação entre os mesmos, quer em termos dos instrumentos utilizados para avaliar o perfecionismo associado às $\mathrm{PA}$, incluindo os seus componentes. Em população não clínica tem sido demonstrada consistentemente a associação entre perfecionismo e comportamento alimentar perturbado (e.g., Minarik \& Ahrens, 1996), bem como níveis elevados de perfecionismo em indivíduos com anorexia e bulimia nervosas (e.g., Bardone-Cone et al., 2007; Bulik et al., 2003). Neste sentido, o perfecionismo tem sido apresentado como um fator de risco para o desenvolvimento de PA (e.g., Shafran, Cooper, \& Fairburn, 2003), apesar de alguns estudos não encontrarem esta associação (e.g., Gustafsson, Edlund, Kjellin, \& Norring, 2009). Por exemplo, Francisco, Alarcão e Narciso (2011) encontraram médias mais elevadas de perfecionismo num grupo de adolescentes com indicação clínica de comportamentos alimentares perturbados, apesar de, na população em geral, o perfecionismo apresentar correlações fracas com esses indicadores.

As formulações teóricas iniciais acerca do perfecionismo são psicodinâmicas e equacionam-no como uma componente neurótica da personalidade. A visão multidimensional deste construto irrompe com a sua operacionalização em duas escalas com o mesmo nome - Multidimentional Perfectionism Scale (MPS) - a MPS-F, de Frost, Marten, Lahart e Rosenblate (1990), e a MPS-H de Hewitt e Flett (1991a), fortemente relacionadas (Frost, Heimberg, Holt, Mattia, \& Neubauer, 1993). O perfecionismo é definido como o desejo de alcançar padrões elevados de desempenho, acompanhado por avaliações pessoais críticas, excessivas e severas em relação ao mesmo (Frost et al., 1990), e reflete tanto componentes de índole pessoal quanto social. A MPS-H compõe-se de três dimensões: 'perfecionismo auto-orientado' (PAO), relacionado com as crenças e expetativas sobre o próprio; 'perfecionismo orientado para os outros' (POO), que remete para as crenças e as expetativas acerca das competências dos outros; e 'perfecionismo socialmente prescrito' (PSP), que pressupõe a perceção da necessidade de lograr padrões irrealistas prescritos por outros significativos que avaliam rigorosamente o indivíduo e que exercem pressão para que seja perfeito. O PSP é mais elevado nas mulheres (Hewitt \& Flett, 1991a) e normalmente associado a psicopatologia mais severa (Frost et al., 1993; Hewitt \& Flett, 1991a), embora alguns autores associem o aumento do risco de psicopatologia à componente autoimposta (e.g., Shafran et al., 2003). Estes opõem-se àqueles que consideram esta componente positiva por si só (e.g., Frost et al., 1993; Stoeber \& Otto, 2006). Quanto à sua origem, o perfecionismo é relacionado por alguns autores com a natureza das relações parentais e consequentes interações (Oliveira, Carmo, Cruz, \& Brás, 2012).

Bento et al. (2010), num estudo com adolescentes portugueses, concluem que níveis elevados de perfecionismo estão associados ao comportamento alimentar perturbado, sendo que a pontuação total da CAPS (Child-Adolescent Perfectionism Scale; Flett, Hewitt, Boucher, Davidson \& Munro, 1992), PAO e PSP surgiram positiva e significativamente associados à pontuação total do EAT (Eating Atitudes Test; Garner, Olmstead, Bohr \& Garfinkel, 1982) em ambos os sexos. Também Macedo et al. (2006), num estudo longitudinal com amostra de estudantes universitários portugueses, encontraram, em ambos os sexos, 
associação entre a pontuação total do MPS-H, do EAT e de todas as dimensões deste. Em raparigas, PAO e PSP foram associados à pontuação total do EAT e em rapazes apenas PSP se revelou preditor da pontuação total do EAT. Considerando ainda as diferenças de género, no estudo de Bento et al. (2010), apenas na dimensão do PAO as raparigas apresentaram resultados médios mais elevados que os rapazes.

\section{Perfecionismo, Ansiedade e Depressão}

A ansiedade e depressão, apontadas como fatores de risco para o desenvolvimento de PA (O'Brien \& Vincent, 2003), são igualmente relacionadas com o perfecionismo. Este último encontra-se associado quer ao aumento de stress (DiBartolo et al., 2007) e à ansiedade em amostras de estudantes e de pacientes psiquiátricos (Hewitt \& Flett, 1991b), como pode consistir um fator predisponente e de manutenção da depressão (e.g., Hewitt \& Flett, 1991b; Kawamura, Hunt, Frost, \& DiBartolo, 2001). Também as diferentes dimensões do perfecionismo se relacionam distintamente com a ansiedade (e.g., Hewitt \& Flett, 1991b) e com a depressão (e.g., Frost et al. 1990, 1993; Sherry, Hewitt, Flett, \& Harvey, 2004), sendo que o PSP parece apresentar frequentemente uma relação mais direta (e.g., Hewitt \& Flett, 1991b), apesar de existirem alguns dados que apontam para PAO quando em interação com acontecimentos de vida stressantes (e.g., Hewitt \& Flett, 1993). Apesar de serem frequentemente encontradas e pesquisadas em conjunto (e.g., González, Cueto, \& Fernández, 2007), alguns autores afirmam que o perfecionismo se associa mais fortemente à sintomatologia depressiva do que à ansiosa, enquanto outros consideram que PAO se associa mais à depressão e PSP mais à ansiedade (Hankin, Roberts, \& Gotlib, 1997). Num estudo de Egan et al. (2013) a ansiedade mediou parcialmente a relação entre PAO e PA numa amostra clínica.

Na adolescência, e não na infância, segundo Nolen-Hoeksema e Girgus (1994), é tido que as raparigas apresentam mais sintomas depressivos e ansiosos (e.g., Derdikman-Eiron et al., 2011), apesar de nem sempre se referirem diferenças em relação à ansiedade (Hankin, 2009; Hankin, Roberts, \& Gotlib. 1997). Visto que a ansiedade é um preditor da depressão mais robusto nas raparigas que nos rapazes (Chaplin, Gillham, \& Seligman, 2009) e que a puberdade desperta mais ansiedade nas raparigas do que nos rapazes (Blumenthal et al., 2011), justifica-se que estas apresentem mais sintomas depressivos. Tal deve-se à experiência de mais desafios desenvolvimentais (Petersen, Sarigani, \& Kennedy, 1991), relacionados com a autoestima, a imagem corporal negativa (Algood-Merten, Lewinsohn, \& Hops, 1990), a insatisfação com o peso, o desenvolvimento de um corpo maduro e a identificação com o papel feminino (Wichstrom, 1999).

\section{PA, Ansiedade e Depressão}

No que diz respeito ao contributo das perturbações emocionais para as PA, diversos estudos com populações clínicas têm apresentado a existência de comorbilidade entre perturbações da ansiedade e/ou depressão e as PA (e.g., O'Brien \& Vincent, 2003) e tal relação é igualmente verificada em adolescentes de ambos os sexos (Eliot \& Baker, 2001) e em amostras não clínicas. Canals, Josepa, Carbajo e Gentzane (1996) apontam o aumento dos níveis de ansiedade como um preditor importante da sintomatologia de PA, sobretudo para as raparigas, indicando que as perturbações de ansiedade podem constituir, assim, um fator de risco para o desenvolvimento destas perturbações (e.g., Kaye et al., 2004). Pode referir-se ainda que o IMC foi identificado como preditor da depressão (Ferreiro et al., 2011) e associado à ansiedade (Crocker et al., 2003) e a sintomas depressivos e ansiosos nas raparigas (Babio et al., 2009). De forma idêntica, os comportamentos de dieta foram relacionados com níveis elevados de depressão e ansiedade (e.g., Pocinho, 2000), sendo mesmo salientado que os adolescentes que realizam dieta devido a depressão se encontram em grande risco de desenvolver PA (Isomaa et al., 2010).

\section{0 presente estudo}

O estudo presente pretende comparar adolescentes de ambos os sexos quanto aos níveis de sintomatologia de comportamento alimentar perturbado, sintomatologia emocional e perfecionismo; comparar adolescentes a efetuar dieta com aqueles que não o fazem; e investigar as relações entre o perfecionismo, a sintomatologia emocional e o comportamento alimentar perturbado em adolescentes portugueses de ambos os sexos.

Considerando a revisão de literatura anteriormente apresentada, estabeleceram-se as seguintes hipóteses: H1) existência, nas raparigas, de níveis superiores de perturbação emocional, sintomatologia depressiva, ansiosa e de perturbação alimentar, bem como maior frequência de comportamento de dieta, comparativamente com os rapazes; H2) ausência de diferenças entre sexos relativamente ao perfecionismo global e suas dimensões auto-orientada e socialmente prescrita; H3) os adolescentes de ambos os sexos que apresentam comportamento de dieta apresentam valores significativamente mais elevados de IMC, perfecionismo e respetivas dimensões, perturbação emocional, sintomatologia 
depressiva, sintomatologia ansiosa e de PA, comparativamente com os adolescentes que não apresentam comportamentos de dieta; H4) o IMC, o perfecionismo (e as suas dimensões auto-orientada e socialmente prescrita), bem como a perturbação emocional, sintomatologia depressiva e ansiosa, são possíveis preditores da sintomatologia de PA, em ambos os sexos.

\section{MÉTODO \\ Participantes}

Participaram no estudo 531 adolescentes (49\% do sexo masculino), estudantes do 3ำ ciclo do ensino básico e secundário de cinco escolas da zona da Grande Lisboa, com idades compreendidas entre os $12 \mathrm{e}$ os 18 anos (rapazes $M=14.79, D P=1.69$; raparigas $M=14.91, D P=1.76$ ). Destes adolescentes, $69 \%$ são provenientes de famílias nucleares intactas, $16 \%$ de família monoparental, $7 \%$ de família reconstruída, $6 \%$ de família transgeracional e $2 \%$ de outras tipologias familiares. Quanto ao nível socioeconómico das mesmas, 48\% usufruem de um nível socioeconómico médio-alto, 32\% de um nível médio e $17 \%$ de um nível baixo. As participantes do sexo feminino apresentam um IMC médio de $20.99(D P=3.13)$ e os participantes do sexo masculino um IMC médio de 21.38 ( $D P=3.67)$.

\section{Instrumentos}

Questionário de Dados Pessoais e Sociodemográficos

0 questionário é composto por um conjunto de itens que se reportam à informação sociodemográfica, como sexo, idade, ano de escolaridade, e a outras variáveis em estudo, nomeadamente peso, altura e comportamentos específicos face à alimentação (medidos através das questões "Faz dieta para emagrecer?" e, em caso de resposta positiva, “Se sim, em que consiste?").

Subescala de perfecionismo do Eating Disorder Inventory 2 (EDI2)

O EDI2 (Garner, 1991) é um instrumento amplo que pretende avaliar atitudes e/ou comportamentos relacionados com a alimentação e que agrega diversas subescalas, entre elas a de perfeccionismo usada neste estudo, constituída por 6 itens de 'perfecionismo' (EDI-P), medida global do traço. Foi utilizada a versão portuguesa do EDI2 de Pocinho (2000; $\alpha=.63$ ), sendo o valor de consistência interna no estudo presente mais elevado $(\alpha=.69)$. De acordo com Enns e Cox (2002), o EDI-P pode ser utilizado como medida breve de perfecionismo no contexto de avaliação das PA, uma vez que raramente foi utilizado fora deste. A revisão de literatura indica, no entanto, a pertinência da distinção entre as componentes intra e interpessoal do mesmo, pelo que considerando a análise de conteúdo dos itens e a experiência de investigações anteriores (Joiner \& Schmidt, 1995; Sherry et al., 2004) considerámos os itens 1, 2 e 4 como relativos à dimensão PSP e os itens 3, 5 e 6 a PAO. Joiner e Schmidt (1995) testaram este procedimento e obtiveram níveis de consistência interna aceitáveis ( $\alpha=.74$ para PAO e $\alpha=.76$ para PSP), e consideraram o modelo a dois fatores mais adequado e estatisticamente superior ao de um fator. Adicionalmente, através de um modelo de equações estruturais, foi demonstrado que, para ambos os sexos, PSP e PAO da EDI-P estão independentemente relacionados com sintomatologia de PA (ver Sherry et al., 2004), o que é favorável à opção pelas duas subdimensões. No presente estudo, os indicadores de consistência interna obtidos, foram mais baixos do que os encontrados por Joiner e Schmidt (1995), mas ainda assim aceitáveis para fins de investigação ( $\alpha=.55$ para PSP e $\alpha=.64$ para PAO).

\section{Eating Attitudes Test-26 (EAT-26)}

O EAT-26 (Garner et al., 1982) identifica três fatores associados ao comportamento alimentar perturbado (dieta, bulimia e preocupação com comida, e controlo oral), a partir das respostas obtidas de população clínica. No presente estudo, apenas foi utilizado o índice global (obtido a partir do somatório do conjunto de 26 itens), à semelhança da maioria dos estudos com população sem referenciação clínica no âmbito das PA (e.g., Dunker \& Philippi, 2003). Este estudo corresponde à versão portuguesa (Francisco, Oliveira, Santos \& Novo, 2011) desta escala, cujo índice global de sintomatologia de PA (comportamento alimentar perturbado) revelou um elevado nível de consistência interna $(\alpha=.91)$.

\section{Cuestionario Educativo-Clínico: Ansiedad y Depresión (CECAD)}

o CECAD (González et al., 2007) avalia a existência de perturbações do tipo ansioso-depressivo, integrando cinco dimensões a que correspondem cinco escalas. A escala de depressão, desenhada para identificar sintomatologia em crianças, adolescentes e jovens adultos, relaciona-se com o humor depressivo ou irritável, insónia ou hipersónia, inutilidade, perda de energia, diminuição da capacidade de pensar, perda de apetite e pensamento suicida. A escala de ansiedade avalia a presença de sintomas psicofisiológicos que lhe estão associados como a tensão muscular e controlo da respiração, medos, preocupações e pensamentos desagradáveis. 0 instrumento integra ainda outras escalas (inutilidade, 
irritabilidade e problemas de pensamento), não utilizadas nesta investigação. As medidas consideradas são relativas às primeiras duas escalas e à medida global do teste, que constitui um indicador de perturbação emocional. Os índices de consistência interna da versão portuguesa (depressão $\alpha=.90$, ansiedade $\alpha=.85$; perturbação emocional $\alpha=.94$ ) de Novo et al. (2006) são semelhantes aos obtidos neste estudo: depressão $\alpha=.93$, ansiedade $\alpha=.90$ e perturbação emocional $\alpha=.96$.

\section{Procedimento}

A recolha de dados foi efetuada nas escolas do $3^{\circ}$ ciclo do ensino básico e secundário após aprovação do projeto pelo Ministério da Educação, autorização das escolas e dos pais dos adolescentes. Os participantes assinaram um consentimento informado, tendo-lhes sido assegurado o anonimato e confidencialidade dos dados. 0 conjunto de instrumentos foi aplicado, num protocolo único, em regime de autoadministração e o tempo de resposta aproximou-se, em média, dos 40 minutos. A aplicação ocorreu em contexto de sala de aula com a presença de um dos investigadores e do professor.

\section{Procedimento de análise de dados}

0 cálculo do IMC foi efetuado com base nos dados acerca do peso e altura reportados pelos participantes. Recorreu-se ao Software PASW Statistics (v. 18.0; SPSS Inc., Chicago, IL) para o tratamento estatístico de dados. Foi assumida a distribuição normal para as variáveis em estudo por se tratar de uma amostra de dimensão razoável, como previsto por Maroco (2010), tendo-se recorrido ao teste de $t$-student para a análise das diferenças de médias em amostras independentes nas principais variáveis em estudo. A variável dieta (dicotómica) foi avaliada com o teste do Qui-quadrado de independência. Foram consideradas estatisticamente significativas as diferenças entre médias cujo $p$-value do teste foi inferior a .05. Por fim, a Regressão Múltipla Hierárquica com seleção de variáveis enter em cada passo foi utilizada para obter um modelo parcimonioso que permitisse predizer a sintomatologia de PA. Utilizou-se o fator de inflação da variância $(V I F)$ para despistar multicolinearidade, pelo que se excluíram as variáveis $P S P$, $P A O$, depressão e ansiedade no sexo masculino e perfecionismo global, PSP, sintomatologia de perturbação emocional, no sexo feminino. Considerou-se para todas as análises uma probabilidade de erro tipo I $(\alpha)$ de .05 .

\section{RESULTADOS}

\section{Hipóteses 1 e 2}

A H1 postula a existência, nas raparigas, de níveis superiores de perturbação emocional, sintomatologia depressiva, ansiosa e de PA, bem como maior frequência de comportamento de dieta, comparativamente com os rapazes. A H2 postula a ausência de diferenças entre sexos relativamente ao perfecionismo global e suas dimensões auto-orientada e socialmente prescrita.

No Quadro 1, apresenta-se a estatística descritiva para as variáveis em estudo, bem como os resultados do teste de diferenças de médias. Estes indicam que as participantes do sexo feminino apresentaram valores médios de perturbação emocional, sintomatologia depressiva, sintomatologia ansiosa e sintomatologia de PA significativamente superiores aos do sexo masculino. Foi ainda reportado um maior número de casos de dieta em indivíduos do sexo feminino $(n=61 ; 22.84 \%)$ comparativamente aos do sexo masculino $(n=28 ; 10.77 \%)$ e, de acordo com o teste do Qui-quadrado, as diferenças observadas são estatisticamente significativas $\left(\chi^{2}(527)=13.7, p<.001\right)$. Estes resultados confirmam a hipótese 1. As diferenças de médias para perfecionismo global, PSP e PAO não se mostraram estatisticamente significativas entre os dois sexos, confirmando a hipótese 2.

Quadro 1. Estatística descritiva das variáveis em estudo e diferenças de médias (N=531).

\begin{tabular}{|c|c|c|c|c|c|c|c|}
\hline \multirow{2}{*}{ Variáveis } & \multirow{2}{*}{ Amplitude } & \multicolumn{2}{|c|}{ Sexo Masculino $(n=261)$} & \multicolumn{2}{|c|}{ Sexo Feminino $(n=270)$} & \multirow{2}{*}{$T$} & \multirow{2}{*}{$\boldsymbol{p}$} \\
\hline & & $M$ & $D P$ & $M$ & $D P$ & & \\
\hline & & & & & & & 1.83 \\
\hline IMC & $0-18$ & 21.38 & 3.67 & 20.99 & 3.13 & 1.34 & .45 \\
\hline Perfecionismo & $0-9$ & 6.87 & 3.80 & 6.61 & 3.91 & 0.75 & .88 \\
\hline PSP & $0-9$ & 4.37 & 2.35 & 4.34 & 2.48 & 0.15 & .39 \\
\hline PAO & $50-250$ & 2.48 & 2.23 & 2.31 & 2.22 & 0.86 & .000 \\
\hline PE & $26-130$ & 91.64 & 24.64 & 108.33 & 28.67 & $-6.58 * * *$ & .000 \\
\hline Depressão & $19-95$ & 48.69 & 13.53 & 57.51 & 15.97 & $-6.47^{* * *}$ & .000 \\
\hline Ansiedade & $0-78$ & 34.12 & 9.99 & 40.08 & 10.82 & $-6.30 * * *$ & .000 \\
\hline PA & & 10.88 & 9.25 & 14.84 & 10.99 & $-4.34 * * *$ & \\
\hline
\end{tabular}

Nota. IMC = índice de massa corporal; $P S P=$ perfecionismo socialmente prescrito; $P A O=$ perfecionismo auto-orientado; $P E=$ perturbação emocional; $P A=$ sintomatologia de perturbação alimentar; ${ }^{* * *} p<.001$ 


\section{Hipótese 3}

H3 sugere que os adolescentes de ambos os sexos que apresentam comportamento de dieta apresentam valores significativamente mais elevados de IMC, perfecionismo e respetivas dimensões, perturbação emocional, sintomatologia depressiva, ansiosa e de PA, comparativamente com os adolescentes que não apresentam comportamentos de dieta.

$\mathrm{Na}$ amostra em estudo, $22.6 \%$ das raparigas $(n=61)$ e $10.7 \%$ dos rapazes $(n=28)$ relatam estar a fazer dieta atualmente. No sexo masculino, quer o IMC quer a sintomatologia de PA são significativamente mais elevados nos adolescentes que fazem dieta $(U=5,031.000, p<.001$ e $U=3,901.500, p<.01$ respetivamente). Este conjunto de resultados apoia parcialmente a hipótese 3, já que relativamente às restantes variáveis em estudo não foram encontradas diferenças significativas. No sexo feminino, o IMC, o perfecionismo global, PAO e sintomatologia de PA são significativamente mais elevados nos adolescentes que fazem dieta (respetivamente: $U=8,818.500, p<.001 ; U=7,138.000, p<.05 ; U=7,707.500, p<.01$; e $U=8,911.500, p<.001)$. Em relação às restantes variáveis em estudo, não foram encontradas diferenças significativas, apoiando apenas parcialmente a hipótese 3.

\section{Hipótese 4}

H4 aponta que o IMC, o perfecionismo (e as suas dimensões auto-orientada e socialmente prescrita), bem como a perturbação emocional, sintomatologia depressiva e ansiosa, são possíveis preditores da sintomatologia de PA, em ambos os sexos.

Dadas as diferenças encontradas na análise da variância entre adolescentes do sexo feminino e masculino, os testes de regressão múltipla hierárquica foram realizados separadamente para cada grupo. De acordo com as hipóteses em estudo e com os dados da literatura, no primeiro passo foram introduzidas as variáveis IMC e dieta. A seleção dos preditores introduzidos no segundo passo teve em conta os valores dos coeficientes de correlação entre a sintomatologia de PA e as três variáveis relacionadas com o perfecionismo (perfecionismo global, PSP e PAO), bem como com as três variáveis relacionadas com a perturbação emocional (perturbação emocional, depressão e ansiedade), encontrados para cada um dos sexos, de forma a evitar situações de multicolinearidade. Assim, para o sexo masculino, relativamente ao perfecionismo selecionou-se o perfecionismo global e, relativamente à perturbação emocional, selecionou-se o indicador geral da mesma, por apresentarem as correlações mais altas, apesar de muito fracas, com a sintomatologia de PA. Para o sexo feminino, relativamente ao perfecionismo, selecionou-se o PAO, e relativamente à perturbação emocional a depressão.

Quadro 2. Resumo da análise de regressão múltipla hierárquica para as variáveis preditoras de PA nos participantes do sexo masculino $(N=261)$.

\begin{tabular}{|c|c|c|c|c|c|c|}
\hline \multirow{2}{*}{ Variável } & \multicolumn{3}{|c|}{ Modelo 1} & \multicolumn{3}{|c|}{ Modelo 2} \\
\hline & $B$ & $S E B$ & $\beta$ & $B$ & $S E B$ & $\beta$ \\
\hline IMC & -0.00 & 0.19 & 0.00 & -0.02 & 0.19 & -0.01 \\
\hline Dieta & 3.75 & 2.47 & 0.11 & 3.84 & 2.38 & 0.11 \\
\hline Perfecionismo global & & & & 0.35 & 0.13 & $0.18^{* *}$ \\
\hline PE & & & & 0.07 & 0.03 & $0.19 * *$ \\
\hline$R^{2}$ & & 0.01 & & & 0.09 & \\
\hline$F$ for change in $R^{2}$ & & 1.20 & & & $4.72 * *$ & \\
\hline
\end{tabular}

Nota. $I M C$ = índice de massa corporal; PE = perturbação emocional (CECAD); **p <.01.

Os resultados da regressão múltipla hierárquica para os indivíduos do sexo masculino indicam que o IMC e a dieta não se relacionam significativamente com a sintomatologia de PA em qualquer dos modelos estudados (Quadro 2). Apenas com a introdução das variáveis perfecionismo e perturbação emocional no terceiro modelo é possível explicar parte da variância de sintomatologia de PA entre os indivíduos do sexo masculino. 0 modelo final permitiu pois explicar $9 \%$ da variância de sintomatologia de PA entre adolescentes do sexo masculino, para a qual contribuem significativamente apenas as variáveis perfecionismo $(\beta=.18, p<.01)$ e perturbação emocional $(\beta=.19, p<.01)$.

Os resultados da regressão múltipla hierárquica para os participantes do sexo feminino indicam que o IMC e a dieta são possíveis preditores de sintomatologia de PA (Quadro 3). No entanto, ao serem introduzidos o PAO e a depressão (Modelo 2), o IMC perde a sua capacidade preditiva, sendo estes em conjunto com a dieta que explicam maior quantidade de variância da variável dependente. 0 modelo final permitiu explicar 38\% da variância de sintomatologia de PA entre as raparigas, com a qual se relacionam sobretudo as variáveis dieta $(\beta=.38, p<.001)$, PAO $(\beta=.17, p<.001)$ e depressão $(\beta=.33, p<.001)$. 0s resultados apoiam a $\mathrm{H} 4$, mas com diferenças entre sexos, uma vez que a sintomatologia de PA surge associada a subdimensões de perfecionismo e de perturbação emocional diferentes em cada grupo. 
Quadro 3. Resumo da análise de regressão múltipla hierárquica para as variáveis preditoras de PA nos participantes do sexo feminino $(N=270)$.

\begin{tabular}{|c|c|c|c|c|c|c|}
\hline \multirow{2}{*}{ Variável } & \multicolumn{3}{|c|}{ Modelo 1} & \multicolumn{3}{|c|}{ Modelo 2} \\
\hline & $B$ & $S E B$ & $\beta$ & $B$ & $S E B$ & $\beta$ \\
\hline IMC & 0.49 & 0.22 & $0.14^{*}$ & 0.23 & 0.20 & 0.07 \\
\hline Dieta & 10.84 & 1.67 & $0.41^{* * *}$ & 10.0 & 1.56 & $0.38 * * *$ \\
\hline PAO & & & & 0.60 & 0.20 & $0.17^{* *}$ \\
\hline Depressão & & & & 0.23 & 0.04 & $0.33^{* * *}$ \\
\hline$R^{2}$ & & 0.23 & & & 0.38 & \\
\hline$F$ for change in $R^{2}$ & & 31.87* & & & $32.63^{* * *}$ & \\
\hline
\end{tabular}

Nota. IMC = índice de massa corporal; PAO = perfecionismo auto-orientado (EDI-P); PE = perturbação emocional (CECAD); ${ }^{*} p<.05,{ }^{* *} p$ $<.01,{ }^{* * *} p<.001$.

\section{DISCUSSÃo}

O presente estudo pretendeu comparar adolescentes de ambos os sexos quanto aos níveis de comportamento alimentar perturbado, sintomatologia emocional e perfecionismo; comparar adolescentes a efetuar dieta com aqueles que não o fazem; e investigar as relações entre IMC, comportamento de dieta, perfecionismo, sintomatologia emocional e comportamento alimentar perturbado em adolescentes portugueses de ambos os sexos.

Observou-se entre os adolescentes participantes, como hipotetizado em $\mathrm{H} 1$, que as raparigas apresentam pontuações mais elevadas do que os rapazes nas escalas de perturbação emocional, depressão e ansiedade, resultado este que reforça as evidências empíricas (e.g., Derdikman-Eiron et al., 2011). Salientamos que as raparigas experienciam a puberdade como mais ansiogénica que os rapazes (Blumenthal et al., 2011) e que sentem mais desafios no desenvolvimento (Petersen et al., 1991) relacionados com a autoestima e imagem corporal negativa (Algood-Merten et al., 1990; Francisco, Narciso, \& Alarcão, 2012), identificação com o papel feminino e com a insatisfação com o peso (Wichstrom, 1999). Também no que toca à sintomatologia de PA, as raparigas exibiram resultados mais elevados que os rapazes, como aliás é relatado diversamente na literatura (e.g., Francisco, Alarcão et al., 2011; Hautala et al., 2007). A confirmação da hipótese não é encarada com surpresa, uma vez que as raparigas apresentaram, da mesma forma, comportamentos de dieta mais frequentes e níveis mais elevados de sinais de depressão e ansiedade, caraterísticas associadas ao desenvolvimento de PA. Verificou-se ainda que o comportamento de dieta era comum, como apontado na literatura (e.g., Patton, 1988), apesar de ter sido mais vezes reportado pelas raparigas do que pelos rapazes, acompanhando o sentido dos dados de outros estudos (e.g., Vaughan \& Halpern, 2010) e indo ao encontro das hipóteses colocadas. Tal pode explicar-se, por exemplo, por uma maior insatisfação com o corpo (Francisco et al., 2012; Rolls et al., 1991), maior tendência para a perceção de excesso de peso (Vaughan \& Halpern, 2010) ou pela tentativa de atingir o ideal corporal de magreza (Davis et al., 2005) que se encontram mais presentes nas raparigas por comparação com os rapazes. Estes, por oposição, tendem a procurar atingir um ideal corporal de muscularidade (Davis et al., 2005), o que pode motivá-los ao exercício físico excessivo (Lewinsohn et al., 2002), aqui não analisado, mais do que aos comportamentos de dieta, justificando, pelo menos parcialmente, as diferenças encontradas.

Em relação às medidas de perfecionismo não foram encontradas diferenças entre os dois sexos, coincidindo com alguns dados da literatura (e.g., Hewitt \& Flett, 1991b) e com a H2, apesar de existirem referências à elevação dos níveis de PSP das mulheres face aos homens (Hewitt \& Flett, 1991a), diferentemente do que havia sido encontrado por Bento et al. (2010), que apontavam para níveis mais elevados de PAO, também nas raparigas. Relativamente ao comportamento de dieta, verificámos que os rapazes e as raparigas com estes comportamentos têm valores médios de IMC superiores. Isto sugere que a dieta estará sobretudo relacionada com a necessidade/desejo de perda de peso por parte destes jovens. Entre os mesmos apuraram-se, de igual modo, valores médios de sintomatologia de PA mais altos, uma relação já descrita em alguns estudos (e.g., Ikeda, 2001) e que vai ao encontro da H3. Não foram, porém, encontradas diferenças no perfecionismo entre rapazes que faziam ou não faziam dieta, infirmando a hipótese elaborada. Esta relação foi, todavia, verificada nas raparigas, confirmando, neste grupo, a hipótese correspondente. Este resultado pode implicar que, apesar de raparigas e rapazes possuírem níveis semelhantes de perfecionismo-traço, este apenas parece ter relevância para o comportamento alimentar perturbado nas raparigas. É possível que tal decorra do facto de as raparigas possuírem um domínio de avaliação perfecionista orientado para o corpo, menos observado nos rapazes, resultante da importância do corpo na autoavaliação das mulheres ocidentais (Stice et al., 2000). Relativamente à sintomatologia depressiva e/ou ansiosa, nem os rapazes nem as raparigas que se encontravam em regime de dieta apresentaram diferenças face àqueles que não se encontravam em dieta, ao contrário do identificado na literatura (Ikeda, 2001) e estipulado na H3. Este resultado pode apontar para a dieta como 
um comportamento "normalizado" na população adolescente, com objetivos bem definidos de controlo da imagem corporal, por exemplo, sem estar necessariamente associado a sintomatologia depressiva ou ansiosa, ao contrário do que acontecerá quando o comportamento de dieta é excessivo e se aproxima do extremo associado às PA.

Somente o perfecionismo global e a perturbação emocional (e não idade, IMC ou dieta) permitiram explicar 9\% da variância de sintomatologia de PA nos rapazes, confirmando apenas parcialmente a H4. Este resultado diverge dos apresentados em revisão, em que as subdimensões do perfecionismo apresentam maior capacidade preditiva (e.g., Macedo et al., 2006). Seria importante analisar o modelo de regressão nos rapazes que efetuam dieta mas devido à dimensão reduzida da amostra, tal não foi possível.

É interessante verificar que o IMC não se revelou estar associado à sintomatologia de PA, quer no modelo de regressão dos rapazes quer das raparigas. Este resultado remete para a importância de outro tipo de variáveis envolvidas no desenvolvimento dos comportamentos alimentares perturbados, especificamente a interiorização do ideal de beleza transmitido pela sociedade ocidental. Neste sentido, será certamente relevante, como referem Francisco et al. (2015) no seu estudo transcultural com adolescentes de Portugal e de Espanha, considerar a interiorização do ideal sociocultural como mediadora da influência do IMC no comportamento alimentar perturbado. Todavia, este papel mediador não foi encontrado para os rapazes de nenhum dos países estudados, reforçando a importância de se considerarem modelos distintos de fatores de risco para o desenvolvimento de PA consoante o sexo dos adolescentes.

Nas raparigas são a dieta, o PAO e a depressão que permitem explicar $38 \%$ da variância de sintomatologia de PA, confirmando parcialmente a hipótese e contrariando estudos anteriores realizados com populações não clínicas. No estudo de Macedo et al. (2006), quer o PAO, quer o PSP se associaram à sintomatologia de PA. Noutros estudos o PAO não se revelou preditor do comportamento alimentar perturbado (Bento et al., 2010) ou apenas o foi quando moderado por outras variáveis, como o PSP (Sherry et al., 2004). Já no estudo de García-Villamisar, Dattilo e Pozo (2012) foi o PSP, e não o PAO, que surgiu como mediador da relação entre afeto depressivo e sintomatologia de PA em estudantes universitárias do sexo feminino.

Os resultados salientam ainda a pertinência da separação das dimensões de perfecionismo, à semelhança do defendido por outros autores, como Joiner e Schmidt (1995), que consideraram o modelo a dois fatores mais adequado e estatisticamente superior ao de um fator, e por Sherry et al. (2004), que alegam que não distinguir os dois tipos de perfecionismo no EDI pode distorcer ou suprimir informação única. 0 papel exato do perfecionismo, como sabemos da revisão literatura, ainda não se encontra plenamente definido, sendo pertinente continuar a estudá-lo, de modo a identificar dimensões específicas relevantes, nomeadamente para a compreensão das PA, tal como defendido por Franco-Paredes et al. (2005).

Algumas limitações ao presente estudo podem ser apontadas, nomeadamente a natureza transversal do estudo, uma vez que não facilita a identificação de relações causais entre as variáveis. Também a opção de agrupar os itens da subescala de Perfecionismo do EDI2 em perfecionismo autoimposto e socialmente prescrito, um método que não foi equacionado pelo autor (Garner, 1991), pode ser indicado como limitação, uma vez que se afasta dos objetivos iniciais da escala e assenta em duas subescalas pouco robustas. Neste sentido, sugere-se que em estudos futuros se utilize um maior número de itens para avaliar as subdimensões do perfecionismo, que parecem estar relacionadas de diferentes formas com os comportamentos alimentares perturbados dos adolescentes.

Todavia, os resultados do presente estudo pretendem ser um contributo para o estudo de características associadas às perturbações alimentares, consideradas na literatura como fatores de risco, confirmando que o fenómeno nos rapazes é ainda pouco conhecido, aludindo indiretamente ao princípio do equifinalidade. Os fatores de risco tradicionalmente apontados para as perturbações alimentares podem ser sobretudo específicos para as raparigas, e não tanto para os rapazes. Tendo em conta os correlatos analisados, futuros estudos longitudinais poderão indicar que o mesmo fim, no caso a sintomatologia de perturbações alimentares, pode ser alcançado através de diferentes caminhos e que estes parecem distanciar-se entre raparigas e rapazes portugueses, aliás, como parece acontecer noutras populações (e.g., Francisco et al., 2015). Seria interessante em estudos futuros aprofundar os diferentes motivos para os adolescentes fazerem dieta e a sua relação com o desenvolvimento posterior de perturbações alimentares. Parece ainda ser necessário conhecer o papel específico do perfecionismo para as perturbações alimentares uma vez que, apesar de não se encontrarem diferenças significativas entre os dois sexos, este relaciona-se de modo diferente com as perturbações alimentares consoante o sexo. De igual modo, talvez o papel específico de perfecionismo auto-orientado nas perturbações alimentares deva ser revisto, pois os dados do presente estudo indicam que este constitui uma vertente mais 
psicopatológica do que o perfecionismo socialmente prescrito, contrariando investigações diversas, fazendo-o sobressair como possível preditor de sintomatologia de perturbação alimentar.

\section{Referências}

American Psychiatric Association. (2002). DSM-IV-TR: Manual de diagnóstico e estatística das perturbações mentais (4⿳亠口了 ed.). Lisboa: Climepsi.

Algood-Merten, B., Lewinsohn, P., \& Hops, H. (1990). Sex differences in adolescent depression. Journal of Abnormal Psychology, 99, 55-63.

Babio, N., Canals, J., Pietrobelli, A., Peréz, S., \& Arija, V. (2009). A two-phase population study: Relationships between overweight, body composition and risk of eating disorders. Nutrición Hospitalaria, 24, 485-491.

Bardone-Cone, A., Wonderlich, S., Frost, R., Bulik, C., Mitchell, J., Uppala, S., \& Simonich, H. (2007). Perfectionism eating disorders: Current status and future directions. Clinical Psychology Review, 27, 384-405.

Bento, C., Pereira, A., Maia, B., Marques, M., Soares, M. J., Bos, S., Valente, J., Gomes, A., Azevedo, M. H. P., \& Macedo, A. (2010). Perfectionism and eating behaviour in Portuguese adolescents. European Eating Disorders Review, 18, 328-337.

Blumenthal, H., Leen-Feldner, E., Babson, K., Gahr, J., Trainor, C., \& Frala, J. (2011). Elevated social anxiety among early maturing girls. Developmental Psychology, 47, 1133-1140.

Bulik, C. M., Tozzi, F., Anderson, C., Mazzeo, S. E., Aggen, S., \& Sullivan, P. F. (2003). The relation between eating disorders and components of perfectionism. The American Journal of Psychiatry, 160, 366368.

Canals, J., Carbajo, J., Fernández, J., Martí-Henneberg, C., \& Domenèch, E. (1996). Biopsychopathology risk profile of adolescents with eating disorder symptoms. Adolescence, 31, 443-450.

Chaplin, T., Gillham, J., \& Seligman, M. (2009). Gender, anxiety and depressive symptoms: A longitudinal study of early adolescents. The Journal of Early Adolescence, 29, 307-327.

Crocker, P., Sabiston, C., Forrestor, S., Kowalski, N., Kowalski, K., \& McDonough, M. (2003). Predicting change in in physical activity, dietary restraint, and physique anxiety in adolescent girls. Canadian Journal of Public Health, 94, 332-337.

Daee, A., Robinson, P., Lawson, M., Turpin, J., Gregory, B., \& Tobias, J. (2002). Psychologic effects of dieting in adolescents. Southern Medical Journal, 95, 1032-1041.

Davis, C., Karvinen, K., \& McCreary, D. (2005). Personality correlates of a drive for muscularity in young men. Personality \& Individual Differences, 39, 349-359.

Derdikman-Eiron, R., Indredavik, M., Bratberg, G., Taraldsen, G., Bakken, I., \& Colton, M. (2011). Gender differences in subjective well-being, self-esteem and psychosocial functioning in adolescents with symptoms of anxiety and depression: Findings from the Nord-Trondelag health study. Scandinavian Journal of Psychology, 52, 261-267.

DiBartolo, P., Li, C., Averett, S., Skotheim, S., Smith, L., Raney, C., \& McMillen, C. (2007). The relationship of perfectionism to judgmental bias and psychopathology. Cognitive Therapy Research, 31, 573-587.

Dunker, K., \& Philippi, S. (2003). Hábitos e comportamentos alimentares de adolescentes com sintomas de anorexia nervosa. Revista de Nutrição, 16, 51-60.

Egan, S., Watson, H., Kane, R., McEvoy, P., Fursland, A., \& Nathan, P. (2013). Anxiety as a mediator between perfectionism and eating disorders. Cognitive Therapy Research, 37, 905-913.

Eliot, A., \& Baker, C. (2001). Eating disordered adolescent males. Adolescence, 36, 535-544.

Enns, M., \& Cox, B. (2002). The nature and assessment of perfectionism: A critical analysis. In G. Flett and P. Hewitt (Eds.), Perfectionism: Theory research, and treatment (pp 33-62). Washington DC: American Psychological Association.

Ferreiro, F., Seone, G., \& Senra, C. (2011). A prospective study of risk factors for the development of depression and disordered eating in adolescents. Journal of Clinical Child and adolescent Psychology, 40, 500-505.

Flett, G., Hewitt, P., Boucher, D., Davidson, L., \& Munro, Y. (1992). The Child-Adolescent Perfectionism Scale: Development, validation and association with adjustment. Department of Psychology Reports (No. 203). Toronto: York University.

Forbush, K., Heatherton, T. F., \& Keel, P. K. (2007). Relationships between perfectionism and specific disordered eating behaviors. International Journal of Eating Disorders, 40, 37-41.

Francisco, R., Alarcão, M., \& Narciso, I. (2011). Avaliação de factores de risco de desenvolvimento de perturbações alimentares: Desenvolvimento e estudos de validação da versão portuguesa do McKnight Risk Factor Survey IV. Revista Iberoamericana de Diagnóstico e Avaliação Psicológica, 32, 143-170. 
Perfecionismo e comportamento alimentar perturbado em adolescentes

Francisco, R., Narciso, I., \& Alarcão, M. (2012). (In)Satisfação com a imagem corporal em adolescentes e adultos portugueses: Contributo para o processo de validação da Contour Drawing Rating Scale. Revista Iberoamericana de Diagnóstico e Avaliação Psicológica, 34, 61-88.

Francisco, R., Oliveira, L., Santos, R., \& Novo, R. (2011). Versão portuguesa do EAT-26 (versão para investigação). Universidade de Lisboa.

Francisco, R., Espinoza, P., González, M., Penelo, E., Mora, M., Rosés, R., \& Raich, R. M. (2015). Body dissatisfaction and disordered eating among Portuguese and Spanish adolescents: The role of individual characteristics and internalisation of sociocultural ideals. Journal of Adolescence, 41, 716.

Franco-Paredes, K., Mancilla-Díaz, J. M., Vázquez-Arévalo, R., López-Aguilar, X., \& Álvarez-Rayón, G. (2005). Perfectionism and Eating Disorders: A Review of the Literature. European Eating Disorders Review, 13, 61-70.

Frost, R., Heimberg, R., Holt, C., Mattia, J., \& Neubauer (1993). A comparison of two measures of perfectionism. Personality and Individual Differences, 14, 119-126.

Frost, R., Marten, P., Lahart, C., \& Rosenblate, R. (1990). The dimensions of perfectionism. Cognitive Therapy and Research, 14, 449-468.

García-Villamisar, D., Dattilo, J., \& Pozo, A. (2012). Depressive mood, eating disorder symptoms, and perfectionism in female college students: A mediational analysis. Eating Disorders, 20, 60-72.

Garner, D. (1991). Eating Disorder Inventory-2 manual. Odessa: Psychological Assessment Resources.

Garner, D., Olmsted, M., Bohr, Y., \& Garfinkel, P. (1982). The Eating Attitudes Test: Psychometric features and clinical correlates. Psychological Medicine, 12, 871-878.

Gonzaléz, L., Cueto, E., \& Fernández, L. (2007). CECAD: Cuestionario educativo-clínico: Ansiedad y depresión. Madrid: Tea Ediciones.

Gustafsson, S., Edlund, B., Kjellin, L., \& Norring, C. (2009). Risk and protective factors for disturbed eating in adolescent girls: Aspects of perfectionism and attitudes to eating and weight. European Eating Disorders Review, 17, 380-389.

Hankin, B. (2009). Development of sex differences in depressive and co-occurring anxious symptoms during adolescence: Descriptive trajectories and potential explanations in a multiwave prospective study. Journal of Child and Adolescent Psychology, 38, 460-472.

Hankin, B., Roberts, J., \& Gotlib, I. (1997). Elevated self-standards and emotional distress during adolescence: Emotional specificity and gender differences. Cognitive Therapy and Research, 21, 663-679.

Hautala, L., Junnila, J., Hlenies, H., Väänänen, A., Liuksila, P., Räihä, H., Välimaki, M., \& Saarijäri (2007). Towards understanding gender differences in disordered eating among adolescents. Journal of Clinical Nursing, 17, 1803-1813.

Hewitt, P., \& Flett, G. (1991a). The multidimensional perfectionism scale: Reliability, validity, and psychometric properties in psychiatric samples. Psychological Assessment: A Journal of Consulting and Clinical Psychology, 3, 464-468.

Hewitt, P., \& Flett, G. (1991b). Perfectionism in the self and social contexts: Conceptualization, assessment and association with psychopathology. Journal of Personality and Social Psychology, 60, 456-470.

Hewitt, P., \& Flett, G. (1993). Dimensions of perfectionism, daily stress, and depression: A test of the specific vulnerability hypothesis. Journal of Abnormal Psychology, 102, 58-65.

Ikeda, J. (2001). Dieting among children and adolescents. Health Weight Journal, 15, 59-64.

Isomaa, K., Isomaa, A., Marttunen, M., Kaltial, A., Heino, R., \& Björkquist, K. (2010). Psychological distress and risk for eating disorders in subgroups of dieters. European Eating Disorders Review, 18, 296303.

Joiner, T., \& Schmidt, N. (1995). Dimensions of perfectionism, life stress, and depressed and anxious symptoms: Prospective support for diathesis-stress but not specific vulnerability among male undergraduates. Journal of Social and Clinical Psychology, 14, 165-183.

Kawamura, K., Hunt, S., Frost, R., \& DiBartolo, P. (2001). Perfectionism, anxiety, and depression: Are the relationships independent? Cognitive Therapy and Research, 25, 291-301.

Kaye, W., Bulik, C., Thornton, L., Barbarich, N., Masters, K., \& Price Foundation (2004). Comorbidity of anxiety disorders with anorexia and bulimia nervosa. American Journal of Psychiatry, 161, 22152221.

Keery, H., van den Berg, P., \& Thompson, J. K. (2004). An evaluation of the Tripartite Influence Model of body dissatisfaction and eating disturbance with adolescent girls. Body Image, 1, 237-251.

Lewinsohn, P. Seeley, J., Moerck, K., \& Striegel-Moore, R. (2002). Gender differences in eating disorder symptoms in young adults. International Journal of Eating Disorders, 32, 426-440. 
Macedo, A., Soares, M., Azevedo, M., Gomes, A., Pereira, A., Maia, B., \& Pato, M. (2007). Perfectionism and eating attitudes in Portuguese university students. European Eating Disorders Review, 15, 296-304.

Maroco, J. (2010). Análise estatística com o PASW Statistics. Pêro Pinheiro: ReportNumber.

Minarik, M., \& Ahrens, A. (1996). Relations of eating behavior and symptoms of depression and anxiety to the dimensions of perfectionism among undergraduate woman. Cognitive Therapy and Research, 20, 155-169.

Nolen-Hoeksema, S., \& Girgus, J. (1994). The emergence of gender differences in depression during adolescence. Psychological Bulletin, 115, 424-443.

Novo, R. F., Lozano, L., Garcia-Cueto, E., Lozano, L. M., Mourão, B., Melo, A. R., Neto, P., Pires, A., \& Faria, M. (2006). A avaliação da Ansiedade e Depressão em contexto escolar: Estudo exploratório da versão portuguesa do CECAD. Poster apresentado na XI Conferência Internacional de Avaliação Psicológica: Formas e Contextos, Braga, Portugal.

O'Brien, K. M., \& Vincent, N. K. (2003). Psychiatric comorbidity in anorexia and bulimia nervosa: Nature, prevalence, and causal relationships. Clinical Psychology Review, 23, 57-74.

Oliveira, D. F., Carmo, C., Cruz, J. P., \& Brás, M. (2012). Perfeccionismo e Representação Vinculativa em Jovens Adultos. Psicologia: Reflexão e Crítica, 25, 514-522.

Patton, G. (1988). The spectrum of eating disorders in adolescence. Journal of Psychosomatic Research, 32, 579-584.

Petersen, A., Sarigani, P., \& Kennedy, R. (1991). Adolescent depression: Why more girls? Journal of Youth and Adolescence, 20, 247-271.

Pocinho, M. (2000). Peso, insatisfação corporal, dietas e patologia alimentar: Um contributo para o estudo das suas relações (Dissertação de mestrado não publicada). Instituto Superior Miguel Torga, Coimbra.

Rolls, B., Fedoroff, I., \& Guthrie, J. (1991). Gender differences in eating behavior and body weight regulation. Health Psychology, 10, 133-142.

Shafran, R., Cooper, Z., \& Fairburn, C. (2003). Clinical perfectionism: A cognitive-behavioural analysis. Behaviour Research and Therapy, 40, 773-791

Sherry, S., Hewitt, P., Besser, A., McGee, B., \& Flett, G. (2004). Self-oriented and socially prescribed perfectionism in the eating disorder inventory perfectionism subscale. International Journal of Eating Disorders, 35, 69-79.

Smink, F., van Hoeken, D., Oldehinkel, A. J., \& Hoek, H. W. (2014). Prevalence and severity of DSM-5 eating disorders in a community cohort of adolescents. International Journal of Eating Disorders, 47, 610619.

Stice, E., Hayhard, C., Cameron, R., Killen, J., \& Taylor, C. (2000). Body image and eating disturbances predict onset of depression among female adolescents: A longitudinal study. Journal of Abnormal Psychology, 109, 438-444.

Stoeber, J., \& Otto, K. (2006). Positive conceptions of perfectionism: Approaches, evidence, challenges. Personality and Social Psychology Review, 10, 295-319.

Striegel-Moore, R. H., \& Bulik, C. M. (2007). Risk factors for eating disorders. American Psychologist, 62, 181-198.

Vaughan, C., \& Halpern, C. (2010). Gender differences in depressive symptoms during adolescence: The contributions of weight-related concerns and behaviors. Journal of Research on Adolescence, 20, 389-419.

Vince, E., \& Walker, I. (2008). A set of meta-analytic studies on the factors associated with disordered eating. Internet Journal of Mental Health, 5, 2-2.

Wichstrom, L. (1999). The emergence of gender differences in depressed mood during adolescence: The role of intensified gender socialization. Developmental Psychology, 35, 232-245. 
Perfecionismo e comportamento alimentar perturbado em adolescentes

Historial do artigo

Recebido 25/09/2014

Aceite $\quad 01 / 04 / 2015$

Publicado 06/2015

Apoio à publicação: Fundação para a Ciência e a Tecnologia (Ministério da Educação e Ciência, Portugal) - Programa FACC 